\title{
Ecodesign na perspectiva do desenvolvimento local e da sustentabilidade
}

Ecodesign in the perspective of local development and sustainability

\section{Écoconception en perspective de développement local et le développement durable}

\author{
Ecodesign en la perspectiva del desarrollo local y de la sostenibilidad \\ Lorene Almeida Tiburtino-Silva ${ }^{1}$ \\ Josemar de Campos Maciel ${ }^{1}$ \\ Reginaldo Brito da Costa ${ }^{1}$ \\ Recebido em 22/05/2017; revisado e aprovado em 18/06/2017; aceito em 18/06/2017 \\ DOI: http://dx.doi.org/10.20435/inter.v19i1.1612

\begin{abstract}
Resumo: O trabalho que segue é um estudo sobre a relação entre ecodesign e desenvolvimento local. Foi realizado a partir de uma varredura de bases de dados de amplo acesso, encontrando textos que desenvolvem questões historiográficas e elaboram categorias de análise e critérios. Como resultado, observa-se que a discussão ainda está em fase de estabilização de critérios e definições e abre perspectivas para a relação entre ecodesign e dinâmicas socioterritoriais.
\end{abstract}

Palavras-chave: design sustentável; potencial do ecodesing; impacto mínimo ao meio ambiente.

Abstract: The work that follows is a study on the relationship between ecodesign and local development. It has been carried out departing from a wide database scan, looking for texts that develop historiographic questions and elaborate categories and analytic criteria. As a result it is observed that the discussion is still in a moment of stabilization of criteria and definitions and opens perspectives for the relation between ecodesign and socio-territorial dynamics.

Keywords: sustainable design; ecodesign potential; minimal impact on the environment.

Résumé: Le travail qui suit est une étude sur la relation entre l'éco et le développement local, réalisée à partir d'une analyse de bases de données. On a trouvé des textes qui développent des problèmes historiographiques et élaborent des critères et catégories d'analyse. En conséquence, on observe que la discussion est encore concentrée sur des critères de stabilisation et définitions. Le travail ouvre des perspectives pour l'étude des relations entre l'écodesign et les dynamiques socio-territoriales.

Mots-clés: design durable; potentiel d'ecoconception; impact minimal sur l'environnement.

Resumen: El trabajo que sigue es un estudio sobre la relación entre el ecodesign y el desarrollo local. Se realizó a partir de una exploración de bases de datos de amplio acceso, encontrando textos que desarrollan cuestiones historiográficas y elaboran categorías de análisis y criterios. Como resultado se observa que la discusión se halla en fase de estabilización de criterios y definiciones. Abrense perspectivas para la relación entre ecodesign y dinámicas socioterritoriales.

Palabras clave: diseño sostenible; diseño potencial; impacto mínimo sobre el medio ambiente.

\section{INTRODUÇÃO}

Há várias conceituações do Desenvolvimento Local em uso. Entre elas, destaca-se a clássica tentativa de Francisco Oliveira (2004), em que ressalta o caráter polissêmico, irredutível a "modelos paradigmáticos". Segundo ele, qualquer tentativa comporta "tantas quantas sejam as dimensões com o intuito do exercício da cidadania" (OLIVEIRA, 2004, p. 13), no quadro de complexidade das reflexões atuais. O livro introdutório de Desai e Porter (2014), por exemplo, ultrapassa os cem capítulos. Naturalmente, uma dessas dimensões é enraizada no contexto da sustentabilidade ambiental.

\footnotetext{
${ }^{1}$ Universidade Católica Dom Bosco (UCDB), Campo Grande, Mato Grosso do Sul, Brasil.
} 
A sustentabilidade é uma das discussões mais importantes do nosso tempo, gerando diversos movimentos de conscientização e construção de ações efetivas, numa busca que provavelmente se propagará por muitas décadas (BARBIERI et al., 2010). Espera-se que seja enraizada e provoque alterações em vários campos de pesquisa, de ações organizacionais e de indivíduos. Podemos dizer que o desenvolvimento local incorpora o esforço da sustentabilidade entre as suas dimensões e esta, por sua vez, atrai várias outras áreas e conceitos, como o ecodesign, que se delineia neste artigo.

O trabalho que segue procura explorar possíveis interações entre o sistema das discussões do desenvolvimento local, combinando ou introduzindo nele algumas referências para delinear a ideia de ecodesign e, ainda, para tentar iluminar algumas das discussões que andam sendo propostas na grande área dos Estudos do Desenvolvimento. Como se pode ver, trata-se de uma busca de interlocuções publicadas em formato de papers, para caracterizar, de forma moderadamente estrutural, a discussão em um estrato da comunidade científica.

\section{METODOLOGIA}

No segundo semestre do ano de 2016, procedeu-se a buscas, privilegiando artigos científicos. As buscas não se restringiram a um intervalo de tempo, tendo sido utilizadas três bases de dados digitais- Google Acadêmico (scholar.google.com.br), Scielo (scielo.br) e Scopus (scopus. com/home.uri). Estas três bases de dados foram selecionadas por sua grande divulgação na comunidade de leitores, além das qualidades específicas que caracterizam cada revista científica e pesquisa em particular que serão citadas logo abaixo.

Para orientar as buscas, foi feita uma leitura de artigos introdutórios à área, sobretudo do texto de Desai e Porter (2014). A partir dessa primeira leitura conceitual informativa, foi delimitado um campo semântico, do qual se extraíram as palavras-chave utilizadas para a busca, relacionando os termos, a saber, "ecodesign e desenvolvimento local", "design for the environment", "design for the environment e local development", "design for the environment e design for sustainability".

Para definir quais artigos seriam considerados válidos, inicialmente foram lidos todos os resumos e as conclusões dos estudos encontrados a partir das palavras-chave. Em seguida, foi realizada uma leitura dos artigos completos que passaram pela triagem inicial. Por meio das referências nos artigos, foram encontrados ainda outros trabalhos. E, após essa varredura, ou screening inicial, a revisão aconteceu em duas etapas: a primeira tenta conceituar o tema, em ordem cronológica e relacionar com o campo conceitual do desenvolvimento local, da sustentabilidade e territorialidade. Em segundo lugar, os artigos foram agrupados de forma a categorizar a reflexão dos autores.

\section{REVISÃO}

\subsection{Origem do tema}

A palavra design é de origem inglesa e foi oficializada em 1588, com a sua inclusão lexical no Dicionário Oxford. Até o momento, design pode ser lido como "a plan or drawing produced to show the look and function or workings of a building, garment, or other object before it is made". Em tradução livre, tem-se um plano ou desenho produzido para mostrar a aparência e a 
função de um objeto. Em uma concepção mais remota, encontra-se o verbo em latim, designo, designare, que abrange o sentido de desenhar (CARDOSO, 2008).

Segundo Naime, Ashton e Hupffer (2012), a palavra design ganhou destaque durante o século XIX, com a revolução industrial. É considerada por Cardoso (2008) como sendo o produto de três processos históricos, a saber: a reorganização das fábricas para produção massiva, a urbanização moderna e a globalização, por meio das redes de comunicação, transportes e comércio.

Porém, a partir do início dos anos setenta, a ideia do desenho fundiu-se parcialmente com uma inspiração oriunda do âmbito ecológico. E, após a leitura dos artigos, é importante notar que é mais comum aparecer o termo ecodesign (MOVILLA et al., 2016) enquanto o termo em inglês, design for environment, aparece em trabalhos advindos da América do Norte (VELEVA et al., 2016). Ainda observaram-se consistirem em sinônimos os termos ecodesign, design verde, design limpo e funcional, ou seja, clean, design sustentável e design para o ambiente. Os resultados similares a esse foram relatados também por Guelere-Filho (2009).

Alguns autores concordam que um momento especial para oficializar a conexão entre design e o conhecimento da ecologia foi a publicação em 1971 do livro "Design for the real World: Human ecology and social change" do designer Victor Papanek (PAZMINO, 2007; CARDOSO, 2008).

No livro, traduzido para 23 idiomas, Papanek (1971) discute a função do design com foco no ser humano, na ética e nos estudos de ecologia. E faz uma crítica ao modelo de estruturação da vida baseado no excesso de consumo, explorando em forma argumentativa a contribuição que os profissionais do design podem fornecer, a fim de diminuir o impacto ambiental. Segundo ele, por exemplo, na fabricação de um objeto, deve-se levar em conta as potencialidades regionais, como o clima e a capacitação pessoal.

É inegável que Papanek (1971) foi inspirado em um discurso mais amplo que contextualiza o seu esforço de refletir sobre a preservação ambiental. No período da publicação de seu livro, os EUA saíam de uma crise do petróleo, e a preocupação com a degradação ambiental começava a tomar dimensões globais. De fato, entre 1970 e 1980, por meio de intensas reflexões, diversas organizações não governamentais ambientalistas terçaram armas numa crítica ao modelo exploratório dos recursos naturais vigentes (CASTELLS, 1999).

Um marco importante para enquadrar esse discurso, foi dado em 1987, com o encontro da Comissão Mundial sobre o Meio Ambiente e Desenvolvimento, também conhecida como Comissão de Brundtland - por ter sido presidida pela norueguesa Gro Harlen Brundtland. Esse encontro tinha como objetivo considerar os avanços da degradação ambiental e o efeito das alternativas adotadas para enfrentá-los (CASTRO, 1996; CAMARGO, 2003).

Entretando, Papanek (1971) também inspirou a evolução do pensamento centrado no ambiente dentro da vertente do design. Para Fiksel (1996), ecodesign é um conjunto de práticas de projeto, dirigidas à concepção de processos e produtos ecoeficientes, com atenção a qualidade ambiental, de saúde e segurança, durante todo o ciclo de vida desses produtos e processos.

Pazmino (2007), em seu artigo "Uma reflexão sobre Design Social, Eco Design e Design Sustentável", faz uma análise qualitativa das diretrizes e conceitos do design social, do ecodesign e do design sustentável apontando suas diferenças e convergências.

A autora define o design social como uma prática que conduza à melhoria da qualidade de vida, renda e inclusão social. Segundo ela, a prática do ecodesign gera um produto competitivo no mercado, cuja produção e uso causem o mínimo de impacto ao meio ambiente, além de possuir formas de mensuração de sua qualidade ambiental. E o design sustentável vai ser, 
então proposto, como um processo mais abrangente e complexo que contemple um produto economicamente viável, ecologicamente correto e socialmente equitativo (PAZMINO, 2007).

Nesse contexto, para Manzini e Vezzoli (2008), o termo ecodesign pode ser definido como: "modelo de projeto pautado por critérios (diretrizes) ecológicos, partindo do enfrentamento das questões ambientais, e até mesmo redesenhar produtos já bem estabelecidos". Na sugestão de Pazmino (2007), algumas diretrizes podem ser lidas no Quadro 1.

- Diretrizes para um projeto pautado por critérios ecológicos:

- Reduzir a utilização de recursos naturais e de energia Usar Materiais não exauríveis (esgotáveis)

- Usar materiais não prejudiciais (danosos e perigosos)

- Usar materiais reciclados, recicláveis e renováveis

- Usar um só material (monomaterial)

- Codificar os materiais para facilitar a sua identificação

- Escolha de técnicas de produção alternativas

- Diminuir os processos produtivos

- Pouca geração de resíduos

- Redução da variabilidade dos produtos

- Reduzir o consumo de energia

- Utilizar tecnologias apropriadas e limpas

- Redução de peso e/ou de volume

- Assegurar a estrutura modular do produto

- Aumentar a confiabilidade e durabilidade

- Eliminar embalagens ou projetar embalagens recicláveis ou reutilizáveis

- Tornar a manutenção e reparos mais fáceis

- Manter os componentes mediante reposições ou refil

- Desmaterializar os produtos (desmontagem para a logística reversa)

Quadro1 - Diretrizes para alcançar os processos do ecodesign.

Fonte: Adaptação de Pazmino (2007).

Além dos critérios acima, há outros, citados por Cerdan et al. (2009), cujos critérios, em geral, apontam para a redução do esgotamento da matéria-prima e outros tipos de impactos ambientais. No trabalho são elencadas oito categorias: redução do número de diferentes materiais e seleção das mais apropriadas; redução do impacto ambiental na fase de produção; otimização da fase de distribuição; redução do impacto ambiental na fase de utilização; extensão da vida útil do produto; simplificação da desmontagem do produto (concepção para desmontagem); concepção para reutilização e design para reciclagem.

No artigo "Sustentabilidade em desenvolvimento de produtos: uma proposta para a classificação de abordagens", Cerdan et al. (2009) delineiam a linha histórica de quinze abordagens próximas ao tema de desenvolvimento de produtos e sustentabilidade ambiental, que ajudam a entender em que contexto histórico se encaixa o termo ecodesign. As abordagens usadas são: Desenvolvimento Sustentável, Balanço de Massa, Ecologia Industrial, Ecoeficiência, Capitalismo Natural, Emissão Zero (ZERI), Berço ao Berço, Cadeia de Suprimentos Verde, Análise do Ciclo de Vida (ACV), EcoDesign, Logística Reversa, Produção Mais Limpa, Design for Environment (DfE), QFD for Environment e Emergia. A Figura 1 adaptada dos autores resume a linha histórica (MAGNAGO; AGUIAR; PAULA, 2012). 


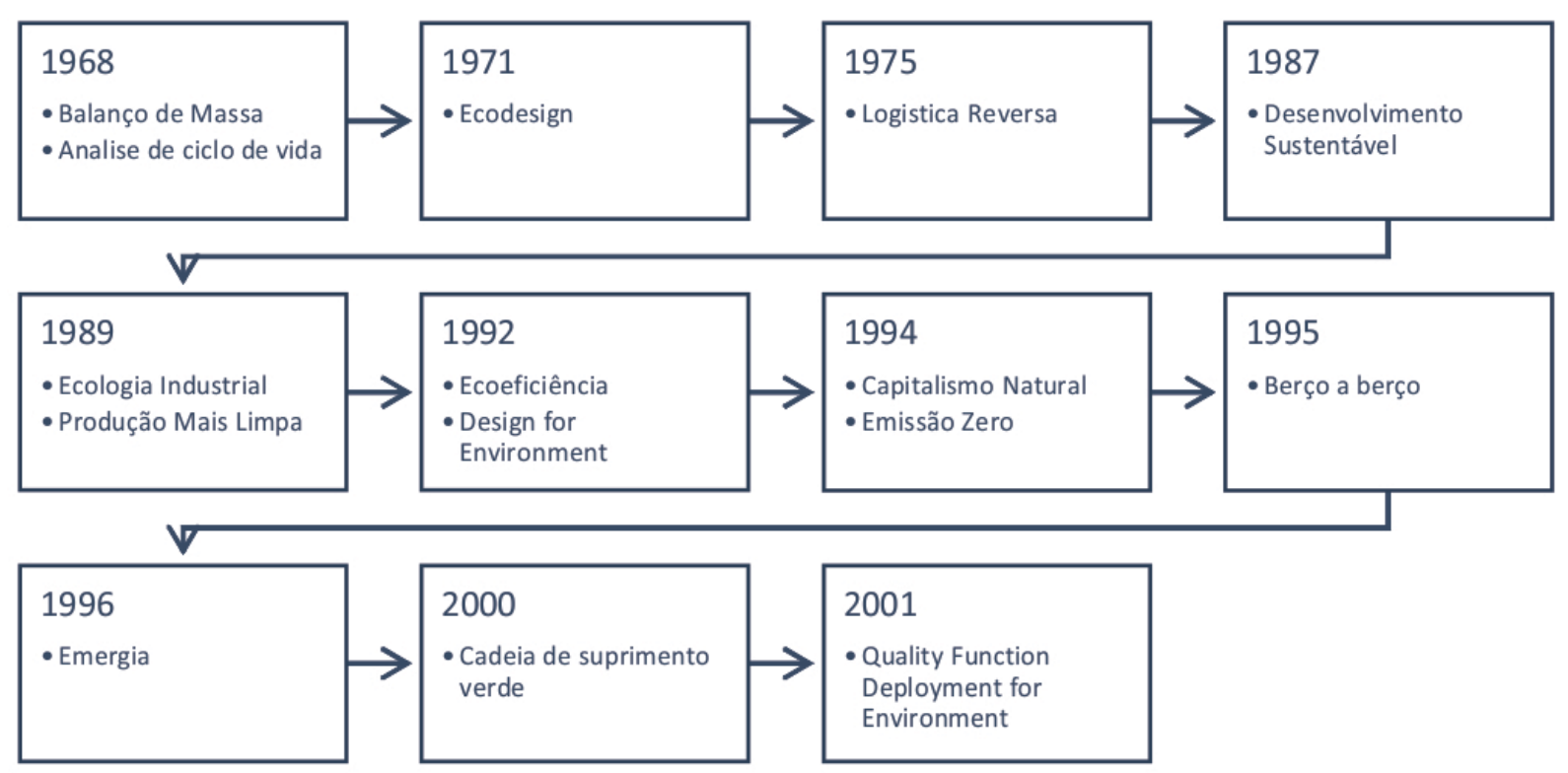

Figura 1 - Linha do tempo dos conceitos relacionados à utilização dos recursos e minimização dos impactos ambientais

Fonte: Adaptado de Magnago, Aguiar e Paula (2012).

Definido, ao menos em parte, o conceito de ecodesign e sua contextualização histórica, é preciso também encontrar autores que fazem uma discussão mais abrangente do tema. Oliveira, Marques e Guedes (2016) falam sobre o design como um valorizador da identidade local, procedendo das transformações ambientais que ocorreram ao longo das últimas décadas, que também influenciaram as áreas do design. Agora espera-se muito mais da relação mercado-produto-marca. Espera-se que haja uma desmaterialização dos objetos e que se proponha uma valorização da cadeia do produto, valorizando seu ambiente de origem ou sua matéria-prima e, em conjunto, a comunidade e a economia local, derivando um conceito muito próximo ao de ecodesign ou de design social.

Um ponto de encontro entre os conceitos de ecodesign e desenvolvimento local pode emergir, da concepção de Desenvolvimento Local, como processo de desenvolvimento cultural e socioeconômico que surge do interior e ganha escalas externas progressivas, situando a comunidade em progressão ascendente de negociações de visibilidade e de ação coletiva (ÁVILA, 2006). Portanto é possível que se estabeleça uma sinergia, com trocas mutuas, onde os processos de ecodesign ajudem na valorização do local e o local alcance o que Ávila (2006) chama de condição de sujeito, e não de objeto participante.

\section{REFERÊNCIAS ILUSTRATIVAS}

Existe uma gama de artigos que, em vez de apresentar ou discutir o conceito, apresentam casos ou iniciativas de variantes do ecodesign, acrescentando nuances e destaques à discussão. Uma amostra dessa diversidade foi elaborada para visualização mais clara no Quadro 2.

Os trabalhos foram categorizados em três grupos, discussão de conceitos, estudos de caso e indicadores para produção de produtos pautados pelo ecodesign. A partir dessa categorização devem emergir reflexões interessantes. 


\begin{tabular}{|c|c|c|c|}
\hline \multicolumn{4}{|c|}{ Discussão de conceitos } \\
\hline Autores & Local & Título & Proposições \\
\hline $\begin{array}{l}\text { Andréia Mesacasa } \\
\text { (2011) }\end{array}$ & Brasil & $\begin{array}{l}\text { Design sustentável e } \\
\text { o desenvolvimento } \\
\text { de produtos com } \\
\text { identidade territorial }\end{array}$ & $\begin{array}{l}\text { Discute a inserção do design sustentável no } \\
\text { desenvolvimento de produtos relacionando } \\
\text { com a valorização da identidade territorial. } \\
\text { A autora destaca que as atividades de } \\
\text { ecodesign podem ser direcionadas para } \\
\text { inúmeras ações que contemplem desde } \\
\text { o tratamento da poluição, passando pela } \\
\text { interferência nos processos produtivos que } \\
\text { geram tal poluição até o redesenho dos } \\
\text { produtos a fim de integrar a perspectiva } \\
\text { ambiental no desenvolvimento de produtos. }\end{array}$ \\
\hline $\begin{array}{l}\text { Andréa Franco } \\
\text { Pereira (2001) }\end{array}$ & Brasil & $\begin{array}{l}\text { Design para a } \\
\text { sustentabilidade: } \\
\text { melhoria de produtos e } \\
\text { processos e valorização } \\
\text { da identidade local. } \\
\end{array}$ & $\begin{array}{l}\text { Trabalha a abordagem de design para a } \\
\text { sustentabilidade, com foco nas necessidades } \\
\text { das pessoas, melhoria do entorno natural, } \\
\text { ganhos econômicos e busca da (re)apropriação } \\
\text { da identidade local. }\end{array}$ \\
\hline $\begin{array}{l}\text { Marcelo Trevisan et } \\
\text { al. (2016) }\end{array}$ & Brasil & $\begin{array}{l}\text { Ecologia industrial, } \\
\text { simbiose industrial e } \\
\text { ecoparque industrial: } \\
\text { conhecer para aplicar }\end{array}$ & $\begin{array}{l}\text { Objetiva fornecer um estudo teórico } \\
\text { conceitual que evidencie a definição, as } \\
\text { características e as escalas de atuação, além } \\
\text { de outros aspectos referentes à Ecologia } \\
\text { Industrial (El), bem como o seu natural vínculo } \\
\text { com o desenvolvimento sustentável. }\end{array}$ \\
\hline $\begin{array}{l}\text { Begum Sertyesilisik } \\
\text { e Egemen } \\
\text { Sertyesilisik (2016) }\end{array}$ & Bélgica & $\begin{array}{l}\text { Eco industrial } \\
\text { development: as a } \\
\text { way of enhancing } \\
\text { sustainable } \\
\text { development }\end{array}$ & $\begin{array}{l}\text { O trabalho analisa como o desenvolvimento } \\
\text { ecoindustrial pode diminuir os danos } \\
\text { ambientais nos processos de produção, } \\
\text { principalmente através do ecodesign (design } \\
\text { para o ambiente). }\end{array}$ \\
\hline \multicolumn{4}{|l|}{ Estudos de caso } \\
\hline $\begin{array}{l}\text { Janaina Ramos } \\
\text { Marcos e Neide } \\
\text { Köhler Schulte } \\
\text { (2009) }\end{array}$ & Brasil & $\begin{array}{l}\text { Ecodesign, } \\
\text { sustentabilidade e o } \\
\text { projeto limonada }\end{array}$ & $\begin{array}{l}\text { Relata a experiência do Projeto Limonada, uma } \\
\text { linha de roupas e acessórios desenvolvidos } \\
\text { a partir da reutilização de calças jeans } \\
\text { descartadas. Com base nos conceitos e } \\
\text { princípios da Sustentabilidade, do Ecodesign, } \\
\text { da reciclagem. }\end{array}$ \\
\hline $\begin{array}{l}\text { Mayara Atherino } \\
\text { Macedo, Eugenio } \\
\text { Andres Diaz Merino } \\
\text { e Maiara Gizelli } \\
\text { Dallazen Camillo } \\
\text { (2014) }\end{array}$ & Brasil & $\begin{array}{l}\text { A gestão de design em } \\
\text { arranjos produtivos } \\
\text { locais (APL): o APL de } \\
\text { móveis do planalto } \\
\text { norte de Santa Catarina }\end{array}$ & $\begin{array}{l}\text { O artigo propõe ações de design que } \\
\text { possam contribuir para o incremento da } \\
\text { competitividade do APL de Móveis do Planalto } \\
\text { Norte Catarinense. } \\
\text { Os autores sugerem o ecodesign como um } \\
\text { design funcional que atua dentro do setor } \\
\text { de APL de produção, tecnologia, materiais, } \\
\text { qualidade e marketing. As ferramentas } \\
\text { usadas para atingir o design funcional, são: } \\
\text { redução dos custos, satisfação do consumidor, } \\
\text { difencial no produto, e maximização da } \\
\text { capacidade de produção. }\end{array}$ \\
\hline $\begin{array}{l}\text { Beatriz Junquera e } \\
\text { Jesús Ángel Del Brío } \\
\text { (2016) }\end{array}$ & Espanha & $\begin{array}{l}\text { Preventive Command } \\
\text { and Control Regulation: } \\
\text { A Case Analysis }\end{array}$ & $\begin{array}{l}\text { Avaliou os efeitos da exigência de } \\
\text { equipamentos pautados no conceito de } \\
\text { ecodesign para a regulamentação ambiental } \\
\text { da União Europeia na fabricação de } \\
\text { automóveis. }\end{array}$ \\
\hline
\end{tabular}




\begin{tabular}{|c|c|c|c|}
\hline \multicolumn{4}{|c|}{ Indicadores norteadores de projeto para ecodesign } \\
\hline Autores & Local & Título & Proposições \\
\hline Chialin Chen (2001) & Canadá & $\begin{array}{l}\text { Design for the } \\
\text { Environment: A Quality- } \\
\text { Based Model for Green } \\
\text { Product Development }\end{array}$ & $\begin{array}{l}\text { O artigo desenvolve um modelo para analisar } \\
\text { as questões estratégicas e políticas relativas ao } \\
\text { desenvolvimento de produtos com atributos } \\
\text { tradicionais e ambientais conflitantes. Foram } \\
\text { analisados aspectos como preferencias } \\
\text { dos 'clientes comuns' e 'clientes verdes', } \\
\text { estratégias do produtor quanto ao número de } \\
\text { produtos, qualidade e preço, e os efeitos dos } \\
\text { padrões ambientais sobre as consequências } \\
\text { econômicas e ambientais do desenvolvimento } \\
\text { de produtos ecológicos. Ao considerar todo } \\
\text { o conjunto, é possível que a pesquisa possa } \\
\text { ser usada para gerenciar e regulamentar o } \\
\text { desenvolvimento de produtos de ecodesign. }\end{array}$ \\
\hline $\begin{array}{l}\text { Carlos Cerdan et al. } \\
\text { (2009) }\end{array}$ & Espanha & $\begin{array}{l}\text { Proposal for new } \\
\text { quantitative eco-design } \\
\text { indicators: a first case } \\
\text { study }\end{array}$ & $\begin{array}{l}\text { O trabalho propõe uma série de indicadores } \\
\text { e testes de ecoconcepção para fornecer } \\
\text { indicadores simples para redução do impacto } \\
\text { ambiental, e assim adequar o projeto para } \\
\text { minimizar os impactos ambientais. }\end{array}$ \\
\hline $\begin{array}{l}\text { FulvioArdente, } \\
\text { Fabrice Mathieux } \\
\text { e Marco Recchioni } \\
\text { (2014) }\end{array}$ & Itália & $\begin{array}{l}\text { Recycling of electronic } \\
\text { displays: Analysis of } \\
\text { pre-processing and } \\
\text { potential ecodesign } \\
\text { improvements }\end{array}$ & $\begin{array}{l}\text { Este artigo analisou a gestão atual e futura } \\
\text { dos eletrônicos e identifica e discute possíveis } \\
\text { recomendações de ecodesign para a sua } \\
\text { melhoria. Com base numa investigação } \\
\text { do tratamento de expositores em duas } \\
\text { instalações de reciclagem europeias típicas, } \\
\text { são identificados os aspectos essenciais } \\
\text { e os aspectos críticos dos métodos de } \\
\text { reciclagem (triagem, desmontagem e pré- } \\
\text { processamento). } \\
\text { O artigo também discute a evolução potencial } \\
\text { de ciclo de vida para eletrônicos e sugere } \\
\text { possíveis recomendações para recicladores, } \\
\text { produtores e gestores políticos para promover } \\
\text { a eficiência de recursos na reciclagem de tais } \\
\text { resíduos. }\end{array}$ \\
\hline $\begin{array}{l}\text { Vesela Veleva et al. } \\
\text { (2016) }\end{array}$ & $\begin{array}{l}\text { Estados } \\
\text { Unidos }\end{array}$ & $\begin{array}{l}\text { Benchmarking } \\
\text { eco-industrial park } \\
\text { development: the case } \\
\text { of Devens }\end{array}$ & $\begin{array}{l}\text { O artigo analisa dados de indicadores de } \\
\text { sustentabilidade no parque ecoindustrial de } \\
\text { Devens-USA. } \\
\text { A maior parte dos progressos foram feitos nas } \\
\text { áreas de transporte, negócios e sustentabilidade } \\
\text { econômica, governança e recursos naturais. Não } \\
\text { sendo observado progressos em questões como } \\
\text { diminuição da geração de resíduos, reciclagem e } \\
\text { melhoria de aterros. }\end{array}$ \\
\hline $\begin{array}{l}\text { Natalia Alonso } \\
\text { Movilla et al. (2016) }\end{array}$ & França & $\begin{array}{l}\text { A method for manual } \\
\text { disassembly analysis to } \\
\text { support the ecodesign } \\
\text { of electronic displays }\end{array}$ & $\begin{array}{l}\text { O objetivo deste artigo foi propor um método } \\
\text { para análise aprofundada de práticas de } \\
\text { desmontagem de telas eletrônicas. Os dados } \\
\text { coletados podem ser usados como evidência } \\
\text { empírica para apoiar o desenvolvimento de } \\
\text { estratégias quantitativas de ecodesign. Alguns } \\
\text { exemplos de estratégias de ecodesign que } \\
\text { podem reduzir significativamente o tempo de } \\
\text { desmontagem da amostra são dados. }\end{array}$ \\
\hline
\end{tabular}

Quadro 2 - Panorama dos artigos com ênfase em Ecodesign e Desenvolvimento Local Sustentável Fonte: Elaborado pelos autores 


\section{CONCLUSÕES}

No momento em que se elabora essa exploração inicial, podem-se ver alguns fenômenos em andamento, que serão sinalizados para, em seguida, apontar possíveis direções da investigação em curso.

O fenômeno mais importante: ainda não aconteceu a estabilização conceitual. O tema do design sustentável ainda se refere à visão do mercado, e sua durabilidade ou viabilidade enquanto empreendimento, e também ao ambiente, e sua conservação como repositório de recursos. Aparece também uma tentativa de relacionar a reutilização de substâncias com a diminuição do impacto sobre o ambiente. Isso são coisas distintas, que podem gerar perspectivas importantes de discussão.

Por último, uma perspectiva de aprofundamento das investigações é o adensamento das informações, ampliando a exploração de bases de dados e suas referências conceituais, bem como a ampliação das discussões.

Como sugestão para futuras pesquisas, principalmente em estudos de caso, deve-se incluir também o aspecto da territorialidade, para além da compreensão dos processos e critérios para execução de um projeto de ecodesign, expandindo a reflexão para o cotidiano e o espaço vivido de envolvidos e interessados.

\section{AGRADECIMENTOS}

À CAPES, pela bolsa concedida.

\section{REFERÊNCIAS}

ARDENTE, F.; MATHIEUX, F.; RECCHION, M. Recycling of electronic displays: Analysis of pre-processing and potential ecodesign improvements. Resources, Conservation and Recycling, v. 92, p. 158-71, 2014. http://dx.doi.org/10.1016/j.resconrec.2014.09.005

ÁVILA, V. F. Realimentando discussão sobre teoria de Desenvolvimento Local (DL). Interações, Campo Grande, MS, v. 8, n. 13, p. 133-40, set. 2006.

BARBIERI, J. C. et al. Inovação e sustentabilidade: novos modelos e proposições. REA - Revista de Administração de Empresas, v. 50, n. 2, p. 146-54, 2010.

CAMARGO, A. L. de B. Desenvolvimento sustentável: dimensões e desafios. Campinas, SP: Papirus, 2003. 160p.

CARDOSO, R. Uma introdução à história do design. 3. ed. São Paulo: Blucher, 2008.

CASTELLS, M. A sociedade em rede. São Paulo: Paz e Terra, 1999.

CASTRO, M. C. Desenvolvimento sustentável. Revista Economia e Empresa, São Paulo, v. 3, n. 3, p. 1-12, 1996.

CERDAN, C.; GAZULLA, C.; RAUGEI, M.; MARTINEZ, E.; PALMES, P. F. Proposal for new quantitative ecodesign indicators: a first case study. Journal of Cleaner Production, v. 17, n. 18, p. 1638-43, dez. 2009.

CHEN, C. Design for the environment: a quality-based model for green product development. Management Sceince, v. 47, n. 2, p. 250-63, 2001.

DESAI, V.; POTTER, R. E. (Ed.). The companion to development studies. 3. ed. London e New York: Taylor and Francis, 2014.

FIKSEL, J. Design for environment. New York: Mc Graw Hill, 1996. 
GUELERE-FILHO, A. Integração do ecodesign ao modelo unificado para a gestão do processo de desenvolvimento de produtos: estudo de caso em uma grande empresa de linha branca. 2009. 242f. Tese (Doutorado em Engenharia de Produção) - Universidade de São Paulo, São Carlos, SP, 2009.

JUNQUERA, B.; BRÍO, J. A. Preventive command and control regulation: a case analsis. Sustainability, v. 8, n. 99, 2016. doi:10.3390/su8010099

OLIVEIRA, I. C. M.; MARQUES, A. M. D. R.; GUEDES, M. G. P. R. Design social para valorização de uma identidade local - design de sistemas sociais. In: INTERNACIONAL FASHION AND DESIGN CONGRESSO, 3. Braga, Portugal: Universidade do Minho, 2016. Disponível em: <http://repositorium.sdum.uminho.pt/ bitstream/1822/41799/1/CIMODE2016_ProceedingsArtigo3.pdf>.

MACEDO, M. A.; MERINO, E. A. D.; CAMILLO, M. G. D. A gestão de design em arranjos produtivos locais (APL): o APL de móveis do planalto norte de Santa Catarina. Iberoamerican Journal of Industrial Engineering, Florianopolis, SC, v. 6, n. 12, p. 1-25, 2014. Disponível em: <http://incubadora.periodicos.ufsc.br/index. php/IJIE/article/view/3151>

MAGNAGO, P. F.; AGUIAR, J. P. O.; PAULA, I. C. Sustentabilidade em desenvolvimento de produtos: uma proposta para a classificação de abordagens. Revista Produção Online, Florianópolis, SC, v. 12, n. 2, p. 351-76, 2012.

MANZINI, E.; VEZZOLI, C. O desenvolvimento de produtos sustentáveis: os requisitos ambientais dos produtos industriais. São Paulo: Edusp, 2008.

MARCOS, J. R.; SCHULTE, N. K. Ecodesign, sustentabilidade e o projeto limonada. Modapalavra e-periódico, v. 2, n. 3, p. 57-70, jan./jul. 2009. Disponível em: <http://www.revistas.udesc.br/index.php/modapalavra/ article/view/7685/5213>.

MESACASA, A. Design sustentável e o desenvolvimento de produtos com identidade territorial. Modapalavra e-periódico, v. 4, n. 8, p. 1-21, jul./dez. 2011. Disponível em: < http://www.revistas.udesc. br/index.php/modapalavra/article/view/7811/5381>.

MOVILLA, N. A.; ZWOLINSKI, P.; DEWULF, J.; MATHIEUX, F. A method for manual disassembly analysis to support the ecodesign of electronic displays. Resources, Conservation and Recycling, v. 114, p. 42-58, nov. 2016. Disponível em: <http://www.sciencedirect.com/science/article/pii/S0921344916301550>.

NAIME, R.; ASHTON, E.; HUPFFER, H. M. Do design ao ecodesign: pequena história, conceitos e princípios. Revista Eletrônica em Gestão, Educação e Tecnologia Ambiental, v. 7, n. 7, p. 1510-9, mar./ago. 2012.

OLIVEIRA, F. Aproximações ao enigma: que quer dizer desenvolvimento local? In: CACCIA-BAVA, Silvio; PAULICS, Veronika; SINK, Peter (Org.). Novos contornos da gestão local: conceitos em construção. São Paulo: Polis, Programa de Gestão Pública e Cidadania/FGV-EAESP, 2004.

PAPANEK, V. Design for the real world - human ecology and social change. Chicago, EUA: Academy Chicago Publishers, 1971.

PAZMINO, A. V. Uma reflexão sobre design social, eco design e design sustentável. In: SIMPÓSIO BRASILEIRO DE DESIGN SUSTENTÁVEL, 1. Curitiba, setembro de 2007. Disponível em: <https://drive.google.com/ file/d/OB-bAfmT1tqbfaTRTazFKVDdtejA/view>.

PEREIRA, A. F. Design para a sustentabilidade: melhoria de produtos e processos e valorização da identidade local. Estudos em Design, Rio de Janeiro, v. 20, n. 2, p.1-15, 2012. Disponível em: <https://www.eed. emnuvens.com.br/design/article/view/98/95>.

SERTYESILISIK, B.; SERTYESILISIK, E. Eco industrial development: as a way of enhancing sustainable development. Journal of Economic Development, Environment and People, Istambul, v. 5, n. 1, 2016. Disponível em: <http://ojs.spiruharet.ro/index.php/jedep/article/view/127/html>.

TREVISAN, M.; NASCIMENTO, F. N.; MADRUGA, L. R. R. G.; NEUTZLING, D. M.; FIGUEIRÓ, P. S.; BOSSLE, M. B. Ecologia industrial, simbiose industrial e ecoparque industrial: conhecer para aplicar. Revista Eletrônica Sistemas \& Gestão, v. 11, n. 2, p. 204-215, 2016. DOI: 10.20985/1980-5160.2016.v11n2.993 
VELEVA, V.; LOWITT, P.; ANGUS, N.; NEELY, D. Benchmarking eco-industrial park development: the case of Devens. Benchmarking: An International Jounal, v. 23, n. 5, p. 1147-70, 2016. Disponível em: <http:// www.emeraldinsight.com/doi/abs/10.1108/BIJ-06-2014-0056>.

\section{Sobre os autores:}

Lorene Almeida Tiburtino-Silva: Doutoranda no programa de mestrado e doutorado em Desenvolvimento Local - Universidade Católica Dom Bosco (UCDB). Bolsista Capes. E-mail: lorenetiburtino@yahoo.com.br

Josemar de Campos Maciel: Programa de Pós-Graduação em Desenvolvimento Local. Universidade Católica Dom Bosco (UCDB), Campo Grande, MS. E-mail: maciel50334@yahoo.com.br

Reginaldo Brito da Costa: Programa de Pós-Graduação em Desenvolvimento Local. Universidade Católica Dom Bosco (UCDB), Campo Grande, MS, Brasil. E-mail: reg.brito.costa@gmail.com 